\title{
Association of Glycated Albumin with Glycemic Markers, Lipid Profile and Liver Function Tests for the Assessment Control in Saudi Patients with Long-Standing Type-2 Diabetes Mellitus
}

\author{
Saifeldeen Babiker Abdalla, Siddig Ibrahim Abelwahab* \\ Department Medical Laboratories, Faculty of Applied Medical Sciences, Jazan University, Saudi Arabia, Department of Biomedical Sciences, Substance \\ Abuse Research Centre, Jazan University, Saudi Arabia.
}

\begin{tabular}{l}
\hline ARTICLE INFO \\
\hline Article history: \\
Received on: $23 / 10 / 2015$ \\
Revised on: $11 / 12 / 2015$ \\
Accepted on: 29/12/2015 \\
Available online: $30 / 03 / 2016$ \\
\hline Key words: \\
Glycated albumin; Diabetes \\
mellitus; Saudi Arabia.
\end{tabular}

\begin{abstract}
This study is an effort to evaluate the association between glycated-albumin and various biochemical parameters in Saudi type-2 diabetic patients. Ninety long-standing (>10 years) type-2 diabetic subjects (51 males, 39 females) serum was analyzed for glycated albumin, fasting blood glucose, hemoglobin1c, cholesterol, triglyceride, albumin, high-density lipoprotein-cholesterol, low-density lipoprotein-cholesterol, aspartate transaminase, alkaline phosphatase, alanine transaminase and total bilirubin. Correlation, principal components analysis, covariance and statistical differences were conducted using SPSS for both male and female participants.

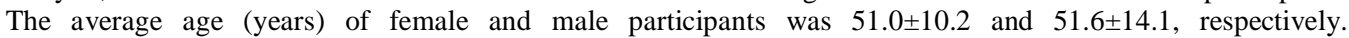
Hemoglobin1C was significantly associated with fasting blood glucose $(r=0.637, P<0.01)$. No significant differences between men and women were observed in the glycemic markers, lipid profile and liver function tests. Both men and women showed no significant differences in glycated-albumin irrespective of age and hemoglobin $1 \mathrm{c}$ covariance. The regression model revealed that low-density lipoprotein, aspartate transaminase and alanine transaminase are significantly associated with glycated-albumin. Men's glycated-albumin was observed to be significantly associated with hemoglobin $1 \mathrm{c}$ only, while women's glycated-albumin is highly associated with low-density lipoprotein only. Glycated-albumin was also co-varied with low-density lipoprotein. Glycated-albumin could be employed for screening high risk diabetic patients for early diagnosis of dyslipidemia and appropriate intervention with lipid-decreasing drugs. Current findings provide novel insights on the use glycated-albumin as clinical chemistry maker.
\end{abstract}

\section{INTRODUCTION}

Diabetes mellitus (DM) is a state of chronic hyperglycemia due to complete or comparative insulin deficiency (Inzucchi et al., 2012). In diabetes, the body either fails to respond to its own insulin, does not make enough insulin or both. This leads to glucose accumulation in the blood that lead to various complications, and includes diabetic ketoacidosis, relative excess of insulin, non ketotic hyper osmolar coma, retinopathy, nephropathy, neuropathy, atherosclerosis, stroke, cardiovascular disease (Forbes and Cooper, 2013; Pollock and Funk, 2013).

The constant hyperglycemia of diabetes is related with continuing harm, dysfunction, and failure of different bodyorgans, especially the kidneys, eyes, heart, nerves, and blood

\footnotetext{
* Corresponding Author

Siddig Ibrahim Abelwahab, Department of Biomedical Sciences, Substance Abuse Research Centre, Jazan University, Saudi Arabia. Email: siddigroa[at]yahoo.com
}

vessels (Liu et al., 2013; Qi et al., 2012). In Type 2 diabetes mellitus (T2DM) the severity of hyperglycemia is enough to cause pathological alterations in different tissues but with unseen clinical signs and during this period it is easy to determine the, abnormalities by measuring fasting blood glucose (Ponchiardi et al., 2013; Seshasai et al., 2011). The diagnostic criteria for DM are blood glucose levels, HbA1C and glycated albumin (GA). Glycated hemoglobin is the word utilized to explain the synthesis of hemoglobin compound formed when a glucose reacts with the amino-group of haemoglobin (Dinu and Moţa, 2014; Lee et al., 2013; Saisho et al., 2011). The hemoglobin molecule binds to the glucose molecule to form a ketoamine (Marigliano et al., 2011). The rate of this reaction is directly related to the plasma glucose. Since a typical red blood cell lives for about 120 days, the HbA1C level at any one time reflects the mean blood glucose levels over previous 60-90 days, therefore measurement of glycated haemoglobin provides a guide for the blood glucose concentration over a period of 3 months (Cohen, 2013; Herman and Cohen, 2012; Kuenen et al., 2011; Lo et al., 2014). 
$\mathrm{HbA} 1 \mathrm{c}$ is a reliable technique of checking long-term diabetes management rather than random test of blood glucose. The normal values of $\mathrm{HbA1C}$ range from 4.5-8.0 $\mathrm{mmol} / \mathrm{L}$ (Wagstaff and Cheung, 2014). The glycated hemoglobin level is affected by the mean glucose levels and RBCs life span so that if RBCs life span reduced, the hemoglobin will have fewer time to turn into glycated form. In this case the glycated hemoglobin test will be less sensitive (Khera et al., 2015).

Glycation is the mechanism that results from binding of a sugar molecule, such as glucose or fructose, to a protein or lipid biomolecule without the need of a catalytic enzyme (Clark et al., 2013). The augmented glucose levels present in DM produces amplified glycation of all proteins, including, albumin (Barlovic et al., 2011). Quantification of the total glycation reaction consequential from the binding of glucose with free amino groups in proteins available in the blood is utilized to screen the level of blood glucose that has normally been present in body fluids over a previous period. Therefore, serum glycated albumin analysis can be employed to examine the present level of glycation of albumin, the main plasma protein (Barlovic et al., 2011; Joseph et al., 2011).

Some limitations were previously reported on the usefulness of HbAlc due to variable lifespan of RBCs and the presence of some disease that decrease the glycation of hemoglobin (Koga, 2014; Raghav and Ahmad, 2014). Therefore, the current study was designed to assess the association between glycated albumin for the assessment control in Saudi patients with long standing T2DM.

\section{MATERIALS AND METHODS}

Quantitative approach of research was adopted, with cross-sectional and analytical design. Samples were collected from different hospitals in Jazan area, Saudi Arabia. Ninety Saudi Arabian long-standing diabetic patients were enrolled in this research. Inclusion criteria were Saudi Arabian patients with diabetes mellitus more than 10 years. Those with diabetes mellitus less than 10 years were excluded. Participants should be with no other chronic diseases.

Permission of this study was obtained from the local authorities in the area of the study. This study was approved by Jazan University Ethical Committee (JUEC-13-15). The aim and benefits of the study were explained to the participant with assurance and confidentiality. An informed consent was collected from all participants. Demographic data such age and gender were collected also. Blood samples were obtained after $12 \mathrm{hrs}$ fasting and kept at $-70^{\circ} \mathrm{C}$ prior to analysis.

\section{Biochemical Analysis}

Blood levels of glucose were measured using spectrophotometric technique. Triglyceride (TG), cholesterol, high density lipoprotein-cholesterol (HDL), low density lipoproteincholesterol (LDL), alanine transaminase (ALT), alkaline phosphatase (ALP), aspartate transaminase (AST) and total bilirubin were analyzed using Hitachi biochemical auto-analyzer (Japan) at Jazan General Hospital, Jazan, Saudi Arabia.

\section{Human glycosylated Albumin}

Glycated albumin (GA) in serum samples was measured according to the instructions provided by available commercial kit (Genasia Biotechnology Co., Ltd, Shanghai, China). Diluted serum and standard were added to GA-antigen pre-coated microtiter plates, gently mixed and left to react for 30 minutes at $37^{\circ} \mathrm{C}$. After washing with washing buffer, HRP-conjugate was added and left to react for 30 minutes at $37^{\circ} \mathrm{C}$ and later washed. TMP-substrate was added and incubated for $10 \mathrm{~min}$ at $37^{\circ} \mathrm{C}$. This blue colored reaction was terminated by the addition of sulphuric acid and the color change (yellow) was detected spectrophotometrically at 450 $\mathrm{nm}$. The concentration of Human GA in the sample was then calculated by comparing the O.D. of the samples to the standard curve.

\section{Glycosylated hemoglobin( HbA1c)}

To eliminate the labile Schiff's base, blood was added to a lysing reagent made by mixing borate ions and detergent. The haemolysate was then added for $5 \mathrm{~min}$ to a soft binding cation exchange resin to allow $\mathrm{HbA} 0$ to be bound with the resin. The resin was then removed using special resin separator to obtain a supernatant fluid with the HbA1. The glycohemoglobin (\%) of total hemoglobin is calculated by the determination of the absorbance of the glycohemoglobin and of the total hemoglobin fraction at $415 \mathrm{~nm}$ in contrast with a standard glycohemoglobin preparation carried through the test procedure.

\section{Statistical analysis}

Collected data were entered, managed and analyzed using SPSS. The mean, SD, frequencies and percentages were obtained. Stepwise linear regression was use for to measure the association of GA and various parameters. $P$-value was obtained to assess the significance of the results. Linear regression was used to analyze the relationship between glycated albumin and various parameters. ANCOVA analysis was used to exclude the main effect of age, as a continuous variable, from the effect of sex on GA levels. Factor analysis using principal components technique was utilized to investigate the covariance of GA and various parameters.

\section{RESULTS AND DISCUSSION}

It is well known that glycation among diverse proteins is augmented in diabetic patients compare with healthy subjects. Some of these glycated proteins are proposed to be linked to the occurrence and development of chronic diabetic complication. Among these glycated proteins, glycated hemoglobin (HbA1C) is frequently used as the gold standard index of glycemic control (Liu et al., 2012; Park et al., 2014; Satheesan et al., 2014). However, HbA1C does not precisely mirror the definite condition of glycemic management in some conditions and patients where 
plasma glucose modifies during short term, and in anemic patients (Mathur et al., 2014). In contrast, another indicator of diabetic control, GA, more precisely reveals alterations in both short term and postprandial plasma glucose levels. Although GA is not affected by ailments of hemoglobin metabolism, it is influenced by ailments of albumin metabolism (Blaak et al., 2012; Zheng et al., 2012). Therefore, the current study was designed to assess the association between GA, glycemic markers, lipid profile and liver function tests for the assessment control in Saudi patients with long standing T2DM.

Ninety long-standing (>10 years) T2DM subjects were included in the study out of which 51 were males and 39 were females. The average age (years) of female and male participants was $51.0 \pm 10.2$ and 51.6 \pm 14.1 , respectively. Table 1 shows the demographic and differences between T2DM male and female in their biochemical parameters, GA and HB1C. The mean value of GA and FBG were higher in males in comparison to female subjects but the differences were non-significant. Kondaveeti et al., (Kondaveeti et al., 2012) was also observed that gender was not a differentiating factor for FBG and GA. ANCOVA analysis was used to exclude the main effect of age, as a continuous variable, from the effect of sex on GA levels. Both men and women showed no significant $(P>0.05)$ differences in GA irrespective of age and $\mathrm{HB} 1 \mathrm{C}$. On the other hand, no significant differences between men and women were observed in HB1C, cholesterol, triglyceride, LDL, HDL, ALT, AST, ALP, total bilirubin and albumin. This suggested that gender was not a differentiating factor in the mentioned parameters when excluding the effect of age. Similar results were also observed previously (Barzin et al., 2012; Webber et al., 2010).

Table 1: Demographic and differences between T2DM male and female in their biochemical parameters, GA and HB1C.

\begin{tabular}{lll}
\hline \multirow{2}{*}{\multicolumn{1}{c}{ Biochemical Parameters }} & \multicolumn{2}{c}{ Biochemical Parameters } \\
\cline { 2 - 3 } & $\begin{array}{l}\text { Female } \\
(\mathbf{N = 3 9 )}\end{array}$ & $\begin{array}{c}\text { Male } \\
(\mathbf{N}=\mathbf{5 1})\end{array}$ \\
\hline Hemoglobin1C (mmol/L) & $9.3 \pm 2.5$ & $9.0 \pm 2.8$ \\
Fasting Blood Glucose (mg/dL) & $188.4 \pm 65.3$ & $191.1 \pm 69.1$ \\
Glycated Albumin (mmol/L) & $55.6 \pm 27.3$ & $59.3 \pm 25.0$ \\
Total Cholesterol (mg/dL) & $206.5 \pm 50.5$ & $195.5 \pm 40.2$ \\
Triglyceride (mg/dL) & $160.4 \pm 36.9$ & $159.6 \pm 35.3$ \\
Low-density lipoprotein-cholesterol (mg/dL) & $47.9 \pm 22.3$ & $50.9 \pm 32.0$ \\
High-density lipoprotein-cholesterol (mg/dL) & $63.6 \pm 17.4$ & $62.5 \pm 13.2$ \\
Alanine transaminase (IU/L) & $29.8 \pm 14.6$ & $32.4 \pm 14.2$ \\
Aspartate transaminase (IU/L) & $30.1 \pm 12.2$ & $31.4 \pm 11.5$ \\
Alkaline phosphatase (IU/L) & $77.3 \pm 18.2$ & $74.1 \pm 19.2$ \\
Total Bilirubin (IU/L) & $0.9 \pm 0.2$ & $0.8 \pm 2$ \\
Albumin (g/dl) & $3.8 \pm 0.4$ & $3.7 \pm 0.3$ \\
\hline
\end{tabular}

*Data showed no significant differences as analyzed by student t-test.

The present study shows no significant $(P>0.05)$ correlation between glycated albumin (GA) and liver function markers, glycemic markers and lipid profile. Hemoglobin $1 \mathrm{C}$ was significantly associated with FBG $(\mathrm{r}=0.637, \mathrm{P}<0.01)$, as shown in Table 2. Bivariate Pearson correlation analysis was followed by backward stepwise linear regression modeling with GA as dependent variable. Surprisingly, the regression model after controlling for some insignificant factors revealed that LDL, ALT and AST are significantly associated with GA (Table 1). The data were further analyzed for male and female separately. Men's GA was observed to be significantly associated with HB1C only, while women's GA is highly associated with LDL only. Although GA is not affected by anemia and variant hemoglobin, it is affected in patients with dysfunction of albumin metabolism (Koga and Kasayama, 2009). GA depicts decreased values in relation to glycemia in patients with hyperthyroidism, nephrotic syndrome and glucocorticoid use in which albumin metabolism elevates. Meanwhile, GA presents superior values comparative to plasma glucose levels in patients with hypothyroidism and hepatic cirrhosis in which albumin metabolism decreases. Certainly, it has been demonstrated that GA was set inferior in relation to plasma glucose levels in hyperuricemic patients, hypertriglyceridemia, smokers and men with non-alcoholic fatty liver disease (NAFLD) with high ALT levels in whom chronic inflammation is suggested (Leite et al., 2009; Targher et al., 2010; Targher et al., 2013).

Table 2: Pearson correlation between, fasting blood glucose and glycated albumin and biochemical parameters.

\begin{tabular}{lccc}
\hline \multicolumn{1}{c}{ Variables } & $\begin{array}{c}\text { Hemoglobin } \\
\text { 1C }\end{array}$ & $\begin{array}{c}\text { Fasting Blood } \\
\text { Glucose }\end{array}$ & $\begin{array}{c}\text { Glycated } \\
\text { Albumin }\end{array}$ \\
\hline Hemoglobin 1C & 1 & $0.637^{* *}$ & -0.134 \\
Fasting Blood Glucose & $0.637^{* *}$ & 1 & -0.116 \\
Glycated Albumin & -0.134 & -0.116 & 1 \\
Cholesterol & 0.007 & -0.017 & -0.010 \\
Triglyceride & 0.000 & -0.078 & 0.013 \\
Low Density Lipoprotein & 0.110 & 0.067 & 0.201 \\
High Density Lipoprotein & -0.105 & -0.010 & -0.015 \\
Alanine Transaminase & -0.037 & 0.100 & 0.012 \\
Aspartate Transaminase & -0.076 & 0.047 & -0.104 \\
Alkaline Phosphatase & 0.005 & 0.001 & -0.080 \\
Total Bilirubin & -0.006 & -0.099 & -0.074 \\
Albumin & 0.089 & $\mathbf{- 0 . 0 6 7}$ & 0.092 \\
\hline Backward Stepwise Linear Regression Model & & \\
\hline Variables included in the model & Beta & $\boldsymbol{t}$ & Sig. \\
\hline (Constant) & & .612 & .542 \\
Low Density Lipoprotein & 0.211 & 2.021 & .046 \\
Alanine Transaminase & 0.458 & 2.252 & .027 \\
Aspartate Transaminase & -0.446 & -2.229 & .029 \\
\hline **significant at 0.01 level of propability. & &
\end{tabular}

Covariation of the study parameters was investigated using factor analysis and principal components analysis (PCA). As shown in Figure 1, five factors were extracted using Varimax method of PCA. $69.13 \%$ of variance was explained by the extract factors. Table 3, shows the distribution of the current study parameters obtained from T2DM patients. GA and LDL were extracted in the same component, with factor loading of 0.575 and 0.773 , respectively (Table 3 ). This confirms that GA was co-varied with LDL. This indicates that GA could be employed for screening high risk diabetic patients for early diagnosis of dyslipidemia and appropriate intervention with hypolipidemic drugs (Karachalias $e t$ al., 2005). It is well known that glycation among a variety of proteins is augmented in diabetic patients compared with healthy subjects. Presently, amongst these glycated proteins, HbA1C is utilized as the gold standard clinical test of glycemic control in clinical settings for diabetes cure (Koga, 2014). However, HbA1C does not precisely show the real standing of glycemic control in some conditions where blood glucose varies throughout short 
term, and in patients who have ailments such as variant hemoglobin and anemia. In contrast, another indicator of clinical glycemic control, GA, more precisely revealed variations in blood glucose during short term and also postprandial plasma glucose. Although GA is not affected by diseases of hemoglobin metabolism, it is influenced by dysfunctions of albumin metabolism (Joseph et al., 2011; Lee et al., 2013; Marigliano et al., 2011; Qi et al., 2012). These include the status of glycemic control changes during short term, diseases which cause iron deficiency anemia, postprandial hyperglycemia, chronic liver disease (liver cirrhosis), chronic renal failure (diabetic nephropathy), pregnancy, and variant hemoglobin. Fasting plasma glucose are altered by numerous factors like stress, acute illness, medication, venous stasis, posture, sample handling, food ingestion, prolonged fasting and exercise. These factors are also likely to affect the $2 \mathrm{hr}$ oral glucose tolerance test. The same factors, however do not have any affects on $\mathrm{HbA1c}$ measurements (Furuya et al., 2014; Speeckaert et al., 2014).

Table. 3: Factor analysis

\begin{tabular}{lccccc}
\hline & \multicolumn{5}{c}{$\begin{array}{c}\text { Number of Components and Factor } \\
\text { Loading }\end{array}$} \\
\cline { 2 - 6 } & 1 & 2 & $\mathbf{3}$ & $\mathbf{4}$ & $\mathbf{5}$ \\
\hline Aspartate Transaminase (AST) & 0.934 & & & & \\
Alanine Transaminase (ALT) & 0.900 & & & & \\
Alkaline Phosphatase (ALP) & 0.612 & & & & \\
Triglyceride & & 0.926 & & & \\
Cholesterol & & 0.916 & & & \\
Hemoglobin 1C (HB1C) & & & 0.895 & & \\
Fasting Blood Glucose (FBG) & & & 0.887 & 0.773 & \\
Low Density Lipoprotein & & & & & \\
Cholesterol (LDL) & & & & 0.575 & \\
Glycated Albumin (GA) & & & & & 0.779 \\
High Density Lipoprotein- - & & & & & 0.610 \\
Cholesterol (HDL) & & & & & 0.489 \\
Albumin & & & & & \\
Total Bilirubin &
\end{tabular}

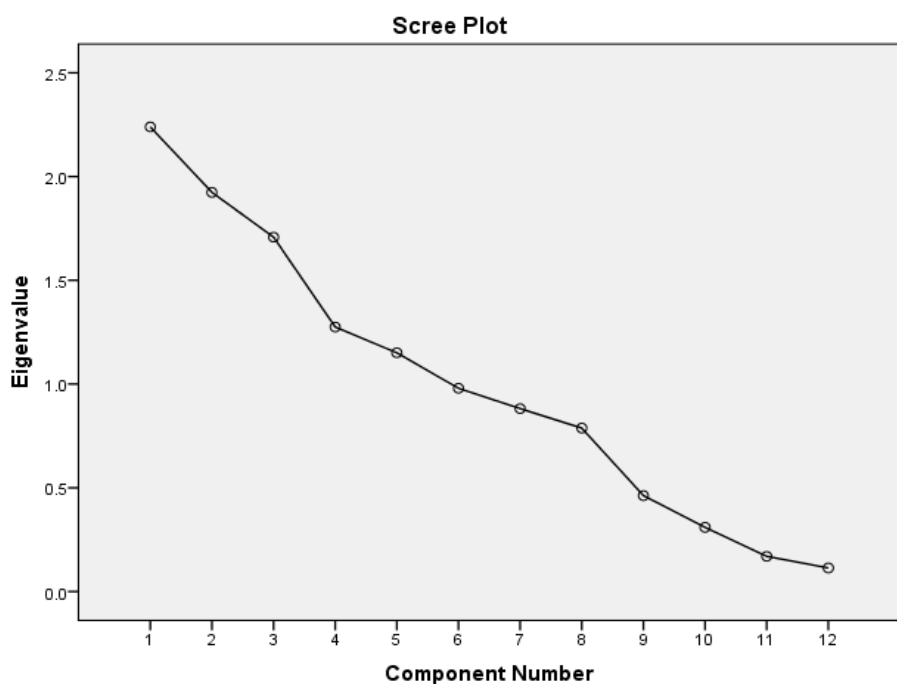

Fig. 1: Scree plot showed the number of factors extracted using factor analysis and principal component analysis.

\section{CONCLUSION AND LIMITATIONS}

The current study is the first of its kind in the Saudi population. Current findings also provide novel insights on the use glycated-albumin as clinical chemistry maker. However, our research had certain boundaries and limitations. Firstly, our subjects were from a uniform population, potentially restraining the generalizability of our findings. Secondly, we did not have information concerning pubescence condition which has helped us to evaluate the effects of pubescence on susceptibility to diabetes, and thirdly, we did not capture into our vision some potential confounders such as nutritional habits, physical activity and socioeconomic condition in our analysis. Additional research is required to establish GA as a better indicator of the diagnosis of DM.

\section{ACKNOWLEDGEMENT}

Deanship of Scientific Research at Jazan University is highly appreciated for funding this research. The project was funded by SABIC Research Fund Scheme (Project No. 33/4/47)

\section{REFERENCES}

Barlovic D, Soro-Paavonen A, Jandeleit-Dahm K RAGE biology, atherosclerosis and diabetes. Clin. Sci., 2011; 121, 43-55.

Barzin M, Hosseinpanah F, Saber H, Sarbakhsh P, Nakhoda K, Azizi $\mathrm{F}$ Gender differences time trends for metabolic syndrome and its components among Tehranian children and adolescents. Cholesterol, 2012; 2012, 12-16.

Blaak E, Antoine JM, Benton D, Björck I, Bozzetto L, Brouns F, Diamant M, Dye L, Hulshof T, Holst J Impact of postprandial glycaemia on health and prevention of disease. Obes. Rev., 2012; 13, 923 984.

Clark SL, Santin AE, Bryant PA, Holman R, Rodnick KJ The initial noncovalent binding of glucose to human hemoglobin in nonenzymatic glycation. Glycobiol, 2013, cwt061.

Cohen MP Clinical, pathophysiological and structure/function consequences of modification of albumin by Amadori-glucose adducts. Bioch. et Biophys. Acta (BBA)-General Subjects, 2013; 1830, 5480-5485.

Dinu I-R, Moța E Glycated Albumin-More Than the Missing Link in the Evaluation of Diabetes Control. Rom J Diabetes Nutr Metab Dis., 2014; 21, 137-150.

Ewa G, Agnieszka P The Tibetan herbal medicines Padma 28 and Padma Circosan inhibit the formation of advanced glycation endproducts (AGE) and advanced oxidation protein products (AOPP) in vitro. BMC Complement. Altern. Med., 2014; 14, 287.

Forbes JM, Cooper ME Mechanisms of diabetic complications. Physiol. Rev., 2013; 93, 137-188.

Furuya A, Suzuki S, Koga M, Oshima M, Amamiya S, Nakao A, Wada K, Okuhara K, Hayano S, Matsuo K HbAlc can be a useful glycemic control marker for patients with neonatal diabetes mellitus older than 20weeks of age. Clin. Chim. Acta, 2014; 436, 93-96.

Herman WH, Cohen RM Racial and ethnic differences in the relationship between $\mathrm{HbAlc}$ and blood glucose: implications for the diagnosis of diabetes. J Clin Endocrinol Metab, 2012; 97, 1067-1072.

Herrick C, Litvin M, Goldberg AC Lipid lowering in liver and chronic kidney disease. Best Pract Res Clin Endocrinol Metab, 2014; 28, 339-352.

Inzucchi SE, Bergenstal RM, Buse JB, Diamant M, Ferrannini E, Nauck M, Peters AL, Tsapas A, Wender R, Matthews DR Management of hyperglycemia in type 2 diabetes: a patient-centered approach position statement of the American Diabetes Association (ADA) and the European Association for the Study of Diabetes (EASD). Diabetes Care, 2012; 35, 1364-1379. 
Joseph K, Anguizola J, Hage DS Binding of tolbutamide to glycated human serum albumin. J. Pharm. Biomed. Anal., 2011; 54, 426432.

Karachalias N, Babaei-Jadidi R, Kupich C, Ahmed N, Thornalley PJ High-Dose Thiamine Therapy Counters Dyslipidemia and Advanced Glycation of Plasma Protein in Streptozotocin-Induced Diabetic Rats. Ann. N. Y. Acad. Sci., 2005; 1043, 777-783.

Khera PK, Smith EP, Lindsell CJ, Rogge MC, Haggerty S, Wagner DA, Palascak MB, Mehta S, Hibbert JM, Joiner CH Use of an oral stable isotope label to confirm variation in red blood cell mean age that influences HbA1c interpretation. Am. J. Hematol., 2015; 90, 50-55.

Koga M Glycated albumin; clinical usefulness. Clin. Chim. Acta, 2014; 433, 96-104.

Koga M, Kasayama S Clinical impact of glycated albumin as another glycemic control marker. Endocr. J., 2009; 57, 751-762.

Kondaveeti SB, Shaker IA, Kumar A, Palwan H Evaluation of glycated albumin and dyslipidemia in type-2 diabetes mellitus. Int.J.Bioassays, 2012; 1, 112-115.

Kuenen JC, Borg R, Kuik DJ, Zheng H, Schoenfeld D, Diamant M, Nathan DM, Heine RJ Does glucose variability influence the relationship between mean plasma glucose and $\mathrm{HbAlc}$ levels in type 1 and type 2 diabetic patients? Diabetes Care, 2011; 34, 1843-1847.

Lee S-Y, Chen Y-C, Tsai I-C, Yen C-J, Chueh S-N, Chuang H$\mathrm{F}$, Wu H-Y, Chiang C-K, Cheng H-T, Hung K-Y Glycosylated hemoglobin and albumin-corrected fructosamine are good indicators for glycemic control in peritoneal dialysis patients. PLoS One, 2013; 8, e57762.

Leite NC, Salles GF, Araujo AL, Villela-Nogueira CA, Cardoso CR Prevalence and associated factors of non-alcoholic fatty liver disease in patients with type-2 diabetes mellitus. Liver Int, 2009; 29, 113-119.

Liu J, Ru Q, Ding Y Glycation a promising method for food protein modification: physicochemical properties and structure, a review. Food Res Int, 2012; 49, 170-183.

Liu T, Gong J, Chen Y, Jiang S Periodic vs constant high glucose in inducing pro-inflammatory cytokine expression in human coronary artery endothelial cells. Inflamm. Res., 2013; 62, 697-701.

Lo C, Lui M, Ranasinha S, Teede HJ, Kerr PG, Polkinghorne KR, Nathan DM, Zheng H, Zoungas S Defining the relationship between average glucose and HbAlc in patients with type 2 diabetes and chronic kidney disease. Diabetes Res. Clin. Pract., 2014; 104, 84-91.

Marigliano M, Casu A, Bertera S, Trucco M, Bottino R Hemoglobin A1C percentage in nonhuman primates: a useful tool to monitor diabetes before and after porcine pancreatic islet xenotransplantation. J Transplant, 2011; 2011.

Mathur S, Zammitt NN, Frier BM Optimal Glycaemic Control in Elderly People with Type 2 Diabetes: What Does the Evidence Say? Drug Saf., 2014, 1-16.

Nin JW, Jorsal A, Ferreira I, Schalkwijk CG, Prins MH, Parving H-H, Tarnow L, Rossing P, Stehouwer CD Higher Plasma Levels of Advanced Glycation End Products Are Associated With Incident Cardiovascular Disease and All-Cause Mortality in Type 1 Diabetes A 12 year follow-up study. Diabetes Care, 2011; 34, 442-447.

Park CH, Noh JS, Tanaka T, Roh S-S, Lee JC, Yokozawa T Polyphenol isolated from Corni Fructus, 7-O-galloyl-d-sedoheptulose, modulates advanced glycation endproduct-related pathway in type 2 diabetic db/db mice. Arch. Pharm. Res., 2014, 1-11.

Pollock F, Funk DC Acute diabetes management: Adult patients with hyperglycemic crises and hypoglycemia. AACN Adv. Crit. Care, 2013; 24, 314-324.

Ponchiardi C, Mauer M, Najafian B Temporal profile of diabetic nephropathy pathologic changes. Current diabetes reports, 2013; $13,592-599$
Qi W-w, Zhong L-y, Li X-r, Li G, Liu Z-x, Hu J-f, Chen N-h Hyperglycemia Induces the Variations of 11. Exp Diabetes Res, 2012; 2012.

Raghav A, Ahmad J Glycated serum albumin: A potential disease marker and an intermediate index of diabetes control. Diabetes \& Metabolic Syndrome: Med. Care Res. Rev, 2014; 8, 245-251.

Rhee CM, Brent GA, Kovesdy CP, Soldin OP, Nguyen D, Budoff MJ, Brunelli SM, Kalantar-Zadeh K Thyroid functional disease: an under-recognized cardiovascular risk factor in kidney disease patients. Nephrol Dial Transplan, 2014, gfu024.

Saisho Y, Tanaka K, Abe T, Shimada A, Kawai T, Itoh H Glycated albumin to glycated hemoglobin ratio reflects postprandial glucose excursion and relates to beta cell function in both type 1 and type 2 diabetes. Diabetol Int, 2011; 2, 146-153.

Satheesan S, Figarola J, Dabbs T, Rahbar S, Ermel R Effects of a new advanced glycation inhibitor, LR-90, on mitigating arterial stiffening and improving arterial elasticity and compliance in a diabetic rat model: aortic impedance analysis. Br. J. Pharmacol., 2014; 171, 3103 3114 .

Seshasai SRK, Kaptoge S, Thompson A, Di Angelantonio E, Gao P, Sarwar N, Whincup PH, Mukamal KJ, Gillum RF, Holme I Diabetes mellitus, fasting glucose, and risk of cause-specific death. N Engl J Med, 2011; 364, 829-841.

Speeckaert M, Van Biesen W, Delanghe J, Slingerland R, Wiecek A, Heaf J, Drechsler C, Lacatus R, Vanholder R, Nistor I Are there better alternatives than haemoglobin A1c to estimate glycaemic control in the chronic kidney disease population? Nephrol Dial Transplan, 2014, gfu006.

Targher G, Bertolini L, Chonchol M, Rodella S, Zoppini G, Lippi G, Zenari L, Bonora E Non-alcoholic fatty liver disease is independently associated with an increased prevalence of chronic kidney disease and retinopathy in type 1 diabetic patients. Diabetol, 2010; 53, 1341-1348.

Targher G, Mantovani A, Pichiri I, Rigolon R, Dauriz M, Zoppini G, Morani G, Vassanelli C, Bonora E Non-alcoholic fatty liver disease is associated with an increased prevalence of atrial fibrillation in hospitalized patients with type 2 diabetes. Clin. Sci., 2013; 125, 301-309.

Wagstaff AE, Cheung NW Diabetes and Hyperglycemia in the Critical Care Setting: Has the Evidence for Glycemic Control Vanished?(Or... Is Going Away?). Current diabetes reports, 2014; 14, 1 10.

Webber M, Krishnan A, Thomas NG, Cheung BM Association between serum alkaline phosphatase and C-reactive protein in the United States National Health and Nutrition Examination Survey 2005-2006. Clin. Chem. Lab. Med., 2010; 48, 167-173.

Zheng C-M, Ma W-Y, Wu C-C, Lu K-C Glycated albumin in diabetic patients with chronic kidney disease. Clin. Chim. Acta, 2012; 413 , $1555-1561$.

How to cite this article:

Abdalla SB, Abelwahab SI. Association of Glycated Albumin with Glycemic Markers, Lipid Profile and Liver Function Tests for the Assessment Control in Saudi Patients with Long-Standing Type-2 Diabetes Mellitus. J App Pharm Sci, 2016; 6 (03): 096-100. 